\title{
A Citrus Huanglongbing Model with Varying Pulse Roguing and General Incidence
}

\author{
Feiping Xie, Shujing Gao*, Dan Yu and Fumin Zhang \\ Key Laboratory of Jiangxi Province for Numerical Simulation and Emulation Techniques, \\ Gannan Normal University, Ganzhou, China \\ Email: gaosjmath@126.com
}

\begin{abstract}
A mathematical model of a grove infected by citrus greening is established, in which an impulsive control strategy of removing infected trees and dead trees is adopted and the general incidence is taken into consideration. By the method of fluctuation, the dynamics behavior is analyzed. Theoretical results show that if $R_{1}<1$ the disease-free periodic solution is global attractive and the disease becomes extinct, if $R_{2}>1$, the disease-free periodic solution is unstable and the disease uniformly persists. By numerical simulation, the theoretical results are illustrated and sensitivity analysis is given.
\end{abstract}

Keywords: Citrus Huanglongbing model, general incidence, global attractivity, permanence.

\section{Introduction}

Citrus Huanglongbing (HLB), previously called citrus greening disease, is one of the most destructive diseases of citrus worldwide, which is characterized by the development of yellow shoots and stunted growth of infected trees combined with a decline in quantity and quality of fruit production [1]. Originally thought to be caused by a virus, it is now known to be caused by unculturable phloem-limited bacteria [2]. HLB is incurable and affects all citrus varieties. HLB bacterium does not pose a threat to humans, pets, or other plants [3]. Now HLB is present in China, eastern and southern Africa, the Indian subcontinent, Madagascar, Mauritius, Reunion, the Saudi Arabian peninsula, and southeast Asia [2]. Because of its impact on many sectors of economy and the implications for the citrus industry nationwide, investigating HLB has become an important project for researchers.

HLB, unlike some other plant diseases, has a incubation period during which the infected trees do not show symptoms. The incubation period is one of the most important parameters influencing HLB within-tree spread [4]. There is ample potential for vectors to acquire the pathogen from trees during the asymptomatic phase of infection [5]. Healthy citrus acquires the pathogen indirectly from both the asymptomatic and the symptomatic infected citrus by vectors.

In the past three decades, compartmental epidemiological models have been developed and they have played an important role in understanding the dynamical behavior of transmission of disease. Recently, several compartmental models on HLB transmission have been investigated and studied $[4,6,7]$. Recently, Chiyaka et al. [4] proposed a model of ordinary differential equations for the HLB transmission dynamics within a tree. Vilamiu et al. $[6,7]$ presented mathematical models for representing the dynamics of HLB disease in a citrus orchard, including the disease's incubation phase in the plants. A HLB model with periodic environment was studied in [8]. The basic reproduction number was given, and some useful comments on controlling the transmission of HLB were proposed by numerical simulation.

Roguing infected trees and dead trees is an important method in the control of HLB. In [9], continuously removing infected and dead trees was considered. In practice, it is an impulsive behavior, and the time intervals vary with the change of seasons. There are various models for the animal disease with impulsive control [10]-[15]. For example, Zhang et al.[13] investigated the dynamic behavior of a predator-prey model with double impulsive control strategy. Xiao et al. [15] made a study of the transmission of West Nile Virus, incorporating a control strategy of culling mosquitoes and investigating its properties. But, for the impulsive control of plant disease, especially for citrus HLB, little work has been done.

Motivated by the above works, our main purpose is to analyze the dynamical behavior theoretically and to study the effects of pulse roguing on the HLB control. To achieve the above goal, we formulate 
impulsive differential equations including susceptible, asymptomatic and symptomatic infected citrus trees, in which the time intervals and the impulsive intensity vary within a year.

The organization of this paper is as follows. In the next section, we propose an impulsive differential system under some hypotheses and the biological interpretation. In Section 3 and Section 4, we obtain the sufficient condition for the global attractivity of the disease-free periodic solution and the permanence of disease, respectively. An example is given in Section 5 in order to illustrate our theoretical results by numerical analysis. A brief conclusion is presented in the last section.

\section{Model Formulation}

Due to the observed delay in the appearance of symptoms of citrus HLB, we subdivide the citrus tree population into three groups: susceptible trees $S$, asymptomatic (latent) infected trees $E$ and symptomatic infected trees $I$. In this paper, we assume that the susceptible individual population is increased via replantation at certain rate in the free space of the grove and diminished by infection which may lead to the increase of group $E$ and $I$ indirectly. Additionally, we consider the disease-associated mortality in the symptomatic citrus population. Because human exploration efficiency to asymptomatic and symptomatic group may be different, we assume they are removed at different rate. Now we establish an impulsive model with general incidence. The system is modeled by the following equations:

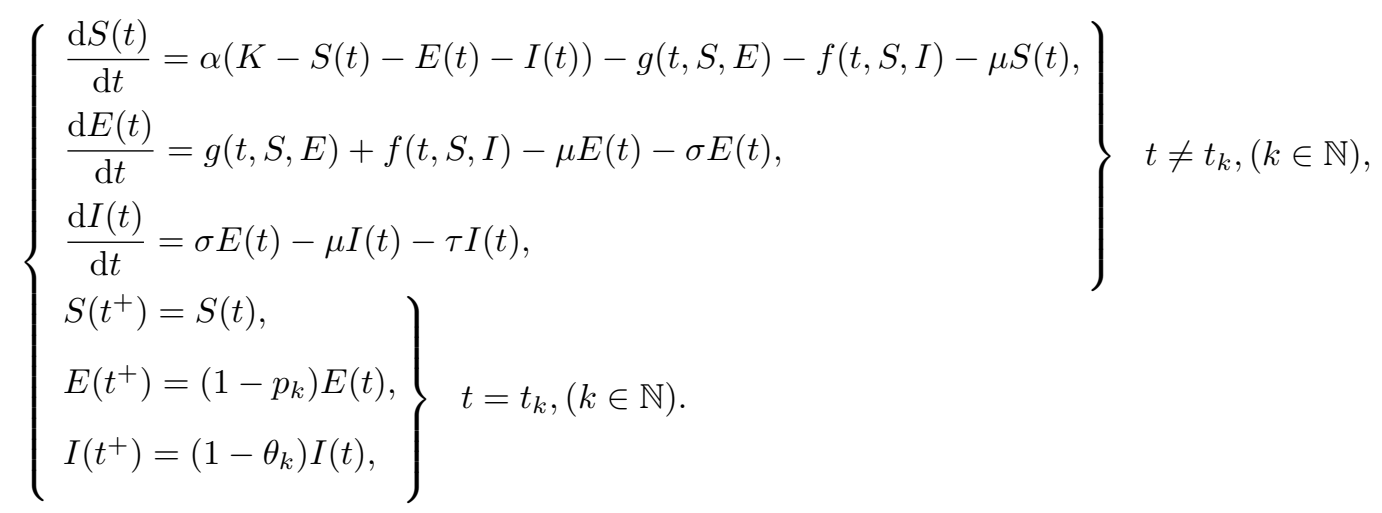

The model is derived from the following assumptions:

- There is a maximum plant population size $K>0$. Recruitment to the population is by replanting at a rate $\alpha>0$ proportional to the difference between the actual number of plants $S+E+I$ and maximum population size $K$.

- $\mu>0$ denotes the natural death rate of citrus, $\tau \geq 0$ is the HLB-induced death rate, $\sigma>0$ represents the transformation rate of infected trees from the latent state to the symptomatic state.

- $S(t), E(t)$ and $I(t)$ are left continuous for $\left[t_{0},+\infty\right)$, that is, $S(t)=\lim _{h \rightarrow 0^{+}} S(t-h), E(t)=\lim _{h \rightarrow 0^{+}} E(t-h)$ and $I(t)=\lim _{h \rightarrow 0^{+}} I(t-h)$.

- $t_{k}$ represent pulse time. There exist a positive integer $q$ and a positive $\omega$ such that $t_{k+q}=t_{k}+\omega$ for all $k \in \mathbb{N}$.

- $p_{k}$ and $\theta_{k}\left(0 \leq \theta_{k}, p_{k} \leq 1\right)$ are the pulse roguing rates of asymptomatic and symptomatic citrus at fixed time $t=t_{k}$, respectively, and $\theta_{k}=\theta_{q+k}, p_{k}=p_{q+k}$ for $k \in \mathbb{N}$.

- Due to the different infectivity of asymptomatic and symptomatic trees, we assume $g(t, S, E)$ and $f(t, S, I)$ represent incidence rates about group $E$ and $I$, respectively. The general nonlinear $g(t, S, E)$ and $f(t, S, I)$ are piecewise continuous, nonnegative, periodic functions with period $\omega$. The form of $g(t, S, E)$ and $f(t, S, I)$ is as follows:

$$
g(t, S, E)=\left\{\begin{array}{c}
g_{1}(t, S, E), \quad t \in\left(t_{0}+n \omega, t_{1}+n \omega\right], \\
\vdots \\
g_{q}(t, S, E), \quad t \in\left(t_{q-1}+n \omega, t_{q}+n \omega\right],
\end{array}\right.
$$




$$
f(t, S, I)=\left\{\begin{array}{cl}
f_{1}(t, S, I), & t \in\left(t_{0}+n \omega, t_{1}+n \omega\right], \\
\vdots & \\
f_{q}(t, S, I), & t \in\left(t_{q-1}+n \omega, t_{q}+n \omega\right],
\end{array}\right.
$$

for all nonnegative integer $n$, and $g_{i}(t, 0, E)=g_{i}(t, S, 0)=0, f_{i}(t, 0, I)=f_{i}(t, S, 0)=0$ for $i=1,2, \cdots, q$.

\section{Global Attractivity of the Disease-Free Periodic Solution}

From system (1), we know that $(\alpha K /(\alpha+\mu), 0,0)$ is the disease-free periodic solution. To analyze the global attractivity of the disease-free periodic solution, we firstly make the following assumption:

$(A)$ : There exist two positive, continuous, periodic functions $\xi_{i}(t), \beta_{i}(t)$ with the period $\omega$, that is $\xi_{i}(t)=\xi_{i}(t+\omega), \beta_{i}(t)=\beta_{i}(t+\omega)$, for all $i=1,2, \cdots, q$, such that $g_{i}(t, S, E) \leq \xi_{i}(t) S(t) E(t), f_{i}(t, S, I) \leq$ $\beta_{i}(t) S(t) I(t)$, for $t \geq t_{0}$.

Theorem 1. If $R_{1}<1$ and system (1) satisfies the assumption (A), then the disease-free periodic solution $(\alpha K /(\alpha+\mu), 0,0)$ is globally attractive, where

$$
R_{1}=\frac{\frac{\alpha K}{\alpha+\mu} \sum_{i=1}^{q} \int_{t_{i-1}}^{t_{i}} i(t) \mathrm{d} t}{\mu \omega-\sum_{i=1}^{q} \ln \left(1-a_{i}\right)},
$$

and

$$
a_{i}=\min \left\{P_{i}, \theta_{i}\right\}, \quad{ }_{i}(t)=\max \left\{\xi_{i}(t), \beta_{i}(t)\right\}, \quad i=1,2, \cdots, q .
$$

Proof. Let $(S(t), E(t), I(t))$ be any solution of system (1). Since $R_{1}<1$, there exists a sufficiently small number $\epsilon_{1}>0$ such that

$$
\Omega \triangleq \exp \left[\sum_{i=1}^{q} \int_{t_{i-1}}^{t_{i}}\left[i(t)\left(\frac{\alpha K}{\alpha+\mu}+\epsilon_{1}\right)\right] \mathrm{d} t+\sum_{i=1}^{q} \ln \left(1-a_{i}\right)-\mu \omega\right]<1 .
$$

From the first equation of (1), we have $\frac{\mathrm{d} S(t)}{\mathrm{d} t} \leq \alpha(K-S(t))-\mu S(t)$. By the comparison theorem, for above mentioned $\epsilon_{1}$, we can obtain that there exists a positive constant $t^{1}\left(>t_{0}\right)$, such that

$$
S(t)<\frac{\alpha K}{\alpha+\mu}+\epsilon_{1}, \text { for all } t \geq t^{1} .
$$

Next, from (4) and the second equation of system (1), we can get that, for $t \in\left(t_{i-1}+n \omega, t_{i}+n \omega\right](i=$ $1,2, \cdots, q)$ and $t \geq t^{1}$

$$
\begin{aligned}
\frac{\mathrm{d} E(t)}{\mathrm{d} t}+\frac{\mathrm{d} I(t)}{\mathrm{d} t} & =g(t, S, E)+f(t, S, I)-\mu(E(t)+I(t))-\tau I(t) \\
& \leq \xi_{i}(t) S(t) E(t)+\beta_{i}(t) S(t) I(t)-\mu(E(t)+I(t)) \\
& \leq \psi_{i}(t) S(t)(E(t)+I(t))-\mu(E(t)+I(t)) \\
& \leq\left[{ }_{i}(t)\left(\frac{\alpha K}{\alpha+\mu}+\epsilon_{1}\right)-\mu\right](E(t)+I(t)),
\end{aligned}
$$

where ${ }_{i}(t)(i=1,2, \cdots, q)$ can be seen in (2). Thus,

$$
\begin{aligned}
& E(t)+I(t) \leq\left(E\left(\left(t_{i-1}+n \omega\right)^{+}\right)+I\left(\left(t_{i-1}+n \omega\right)^{+}\right)\right) \exp \int_{t_{i-1}+n \omega}^{t}\left[i(t)\left(\frac{\alpha K}{\alpha+\mu}+\epsilon_{1}\right)-\mu\right] \mathrm{d} t \\
& =\left[\left(1-p_{i-1}\right) E\left(t_{i-1}+n \omega\right)+\left(1-\theta_{i-1}\right) I\left(t_{i-1}+n \omega\right)\right] \exp \int_{t_{i-1}+n \omega}^{t}\left[i(t)\left(\frac{\alpha K}{\alpha+\mu}+\epsilon_{1}\right)-\mu\right] \mathrm{d} t \\
& \leq\left(1-a_{i-1}\right)\left(E\left(t_{i-1}+n \omega\right)+I\left(t_{i-1}+n \omega\right)\right) \exp \int_{t_{i-1}+n \omega}^{t}\left[i(t)\left(\frac{\alpha K}{\alpha+\mu}+\epsilon_{1}\right)-\mu\right] \mathrm{d} t,
\end{aligned}
$$


where $a_{i}(i=1,2, \cdots, q)$ are defined in (2).

By using the similar method, we can deduce from (5) that for $t \in\left(t_{i-1}+n \omega, t_{i}+n \omega\right]$

$$
\begin{aligned}
E(t)+I(t) & \leq \prod_{j=1}^{i-1}\left(1-a_{j}\right)\left[E\left(\left(t_{0}+n \omega\right)^{+}\right)+I\left(\left(t_{0}+n \omega\right)^{+}\right)\right] \\
& \times \exp \left\{\left(\frac{\alpha K}{\alpha+\mu}+\epsilon_{1}\right)\left[\int_{t_{0}+n \omega}^{t_{1}+n \omega} \psi_{1}(t) \mathrm{d} t+\cdots+\int_{t_{i-1}+n \omega}^{t} i(t) \mathrm{d} t\right]-\mu\left(t-t_{0}-n \omega\right)\right\} .
\end{aligned}
$$

Especially, when $t=t_{0}+(n+1) \omega$, we have

$$
\begin{aligned}
& E\left(\left(t_{0}+(n+1) \omega\right)^{+}\right)+I\left(\left(t_{0}+(n+1) \omega\right)^{+}\right) \\
& \leq\left(1-a_{q}\right)\left(E\left(t_{q}+n \omega\right)+I\left(t_{q}+n \omega\right)\right) \\
& \leq \prod_{i=1}^{q}\left(1-a_{i}\right)\left[E\left(\left(t_{0}+n \omega\right)^{+}\right)+I\left(\left(t_{0}+n \omega\right)^{+}\right)\right] \exp \left[\sum_{i=1}^{q} \int_{t_{i-1}}^{t_{i}}\left[i(t)\left(\frac{\alpha K}{\alpha+\mu}+\epsilon_{1}\right)\right] \mathrm{d} t-\mu\left(t_{q}-t_{0}\right)\right] \\
& =\left[E\left(t_{0}+n \omega\right)+I\left(t_{0}+n \omega\right)\right] \exp \left[\sum_{i=1}^{q} \int_{t_{i-1}}^{t_{i}}\left[i(t)\left(\frac{\alpha K}{\alpha+\mu}+\epsilon_{1}\right)\right] \mathrm{d} t+\sum_{i=1}^{q} \ln \left(1-a_{i}\right)-\mu \omega\right] \\
& =\Omega\left[E\left(\left(t_{0}+n \omega\right)^{+}\right)+I\left(\left(t_{0}+n \omega\right)^{+}\right)\right] .
\end{aligned}
$$

Therefore, for any positive integer $s$, we obtain the following result: $E\left(\left(t_{0}+(n+s) \omega\right)^{+}\right)+I\left(\left(t_{0}+(n+s) \omega\right)^{+}\right) \leq$ $\Omega^{s}\left[E\left(\left(t_{0}+n \omega\right)^{+}\right)+I\left(\left(t_{0}+n \omega\right)^{+}\right)\right]$, combining with (3), we get

$$
E\left[\left(t_{0}+(n+s) \omega\right)^{+}\right]+I\left[\left(t_{0}+(n+s) \omega\right)^{+}\right] \rightarrow 0, \text { as } s \rightarrow+\infty .
$$

According to (6) and (7), we have

$$
\lim _{t \rightarrow+\infty}(E(t)+I(t))=0,
$$

that is, for above mentioned $\epsilon_{1}$, there exists $t^{2}\left(>t^{1}\right)$, we get $E(t)+I(t)<\epsilon_{1}$ for all $t>t^{2}$.

Then, from the first equation of system (1), we know for $t>t^{2}$,

$$
\begin{aligned}
\frac{\mathrm{d} S(t)}{\mathrm{d} t} & =\alpha(K-S(t)-E(t)-I(t))-g(t, S, E)-f(t, S, I)-\mu S(t) \\
& \geq \alpha\left(K-\epsilon_{1}\right)-\left(\psi^{*} \epsilon_{1}+\alpha+\mu\right) S(t),
\end{aligned}
$$

where $\psi^{*}=\max _{1 \leq i \leq q}\left\{\psi_{i}(t), t \in\left[t_{0}, t_{0}+\omega\right]\right\}$. Solving the differential inequality, we can obtain

$$
S(t) \geq \frac{\alpha\left(K-\epsilon_{1}\right)}{\psi^{*} \epsilon_{1}+\alpha+\mu}+\left[S\left(t^{2}\right)-\frac{\alpha\left(K-\epsilon_{1}\right)}{\psi^{*} \epsilon_{1}+\alpha+\mu}\right] e^{-\left(t-t^{2}\right)\left(\psi^{*} \epsilon_{1}+\alpha+\mu\right)} \triangleq \tilde{S}(t), \text { for } t>t^{2} .
$$

It follows from (4) and (10) that

$$
\tilde{S}(t) \leq S(t) \leq \frac{\alpha K}{\alpha+\mu}+\epsilon_{1}, \text { for }\left(t>t^{2}\right) .
$$

Because $\epsilon_{1}$ is an arbitrary positive number, (10) means that

$$
\lim _{t \rightarrow+\infty} S(t)=\frac{\alpha K}{\alpha+\mu} .
$$

By (8) and (11), we get that the disease-free periodic solution $(\alpha K /(\alpha+\mu), 0,0)$ is globally attractive.

\section{Permanence}

In this section, we mainly obtain the sufficient conditions for the permanence of system (1). We give the following assumption at first.

$(B)$ : There exist two positive, continuous, periodic functions $\varsigma_{i}(t), \eta_{i}(t)$ with period $\omega$, such that $g_{i}(t, S, E) \geq \varsigma_{i}(t) S(t) E(t), f_{i}(t, S, I) \geq \eta_{i}(t) S(t) I(t)$, for $t \geq t_{0}$. 
Theorem 2. If $R_{2}>1$ and assumptions $(A)$ and $(B)$ hold, then system (1) is permanent, where

$$
R_{2}=\frac{\frac{\alpha K}{\alpha+\mu} \sum_{i=1}^{q} \int_{t_{i-1}}^{t_{i}} \varphi_{i}(t) \mathrm{d} t}{(\mu+\tau) \omega-\sum_{i=1}^{q} \ln \left(1-b_{i}\right)},
$$

and

$$
b_{i}=\max \left\{p_{i}, \theta_{i}\right\}, \varphi_{i}(t)=\min \left\{\varsigma_{i}(t), \eta_{i}(t)\right\}, \quad i=1,2, \cdots, q .
$$

Proof. Since $R_{2}>1$, there exists a sufficiently small number $\epsilon_{2}>0$ such that

$$
\Sigma \doteq \prod_{i=1}^{q}\left(1-b_{i}\right) \exp \left[\left(\frac{\alpha\left(K-\epsilon_{2}\right)}{\alpha+\mu}-\epsilon_{2}\right) \sum_{i=1}^{q} \int_{t_{i-1}}^{t_{i}} \varphi_{i}(t) \mathrm{d} t-(\mu+\tau) \omega\right]>1 .
$$

In order to illustrate the conclusion, we firstly prove the disease is uniformly weakly persistent, that is to say, there exists a positive constant $\eta>0$, such that $\limsup _{t \rightarrow+\infty}(E(t)+I(t)) \geq \eta$. By contradiction, for above given $\epsilon_{2}>0$, there exists a constant $t^{3}>0$ such that $E(t)+I(t)<\epsilon_{2}$ for all $t>t^{3}$.

According to assumption (A) and the first equation of system (1), we know

$$
\begin{aligned}
\frac{\mathrm{d} S(t)}{\mathrm{d} t} & =\alpha(K-S(t)-E(t)-I(t))-g(t, S, E)-f(t, S, I)-\mu S(t) \\
& \geq \alpha\left(K-\epsilon_{2}\right)-\left(\psi^{*} \epsilon_{2}+\alpha+\mu\right) S(t), \quad \text { for all } t>t^{3},
\end{aligned}
$$

where $\psi^{*}$ is defined in (9). By comparison theorem, we have $S(t) \geq y_{1}(t)$ and $y_{1}(t) \rightarrow \frac{\alpha\left(K-\epsilon_{2}\right)}{\psi^{*} \epsilon_{2}+\alpha+\mu}$ as $t \rightarrow+\infty$, where $y_{1}(t)$ is the solution of the following comparison system:

$$
\frac{\mathrm{d} y_{1}(t)}{\mathrm{d} t}=\alpha\left(K-\epsilon_{2}\right)-\left(\psi^{*} \epsilon_{2}+\alpha+\mu\right) y_{1}(t) .
$$

Therefore, for above mentioned $\epsilon_{2}$, there exists an integer $n^{*}>0$ such that

$$
S(t) \geq y_{1}(t) \geq \frac{\alpha\left(K-\epsilon_{2}\right)}{\psi^{*} \epsilon_{2}+\alpha+\mu}-\epsilon_{2} \quad \text { for all } t>t^{3}+n^{*} \omega .
$$

For above mentioned $t^{3}+n^{*} \omega$, we can get that there exists a positive integer $N_{1}$, such that $N_{1} \omega \geq$ $t^{3}+n^{*} \omega$. Hence for all $n \omega+t_{i-1}<t<n \omega+t_{i},\left(n \geq N_{1}, i=1,2, \cdots, q\right)$, by the second equation of system (1) and (14), we have

$$
\begin{aligned}
\frac{\mathrm{d} E(t)}{\mathrm{d} t}+\frac{\mathrm{d} I(t)}{\mathrm{d} t} & =g(t, S, E)+f(t, S, I)-\mu(E(t)+I(t))-\tau I(t) \\
& \geq \varsigma_{i}(t) S(t) E(t)+\eta_{i}(t) S(t) I(t)-\mu(E(t)+I(t))-\tau I(t) \\
& \geq \varphi_{i}(t) S(t)(E(t)+I(t))-\mu(E(t)+I(t))-\tau(E(t)+I(t)) \\
& \geq\left[\varphi_{i}(t)\left(\frac{\alpha\left(K-\epsilon_{2}\right)}{\psi^{*} \epsilon_{2}+\alpha+\mu}-\epsilon_{2}\right)-\mu-\tau\right](E(t)+I(t)),
\end{aligned}
$$

where $\varphi_{i}(t)=\min \left\{\varsigma_{i}(t), \eta_{i}(t)\right\}(i=1,2, \cdots, q)$ are defined in (12). In addition, in view of system (1), we yield

$$
E\left(t^{+}\right)+I\left(t^{+}\right)=\left(1-p_{i}\right) E(t)+\left(1-\theta_{i}\right) I(t) \geq\left(1-b_{i}\right)((E(t)+I(t)),
$$

where $b_{i}=\max \left\{p_{i}, \theta_{i}\right\},(i=1,2, \cdots, q)$ can be seen in (12).

Then we consider impulsive comparison system:

$$
\left\{\begin{array}{l}
\frac{\mathrm{d} y_{2}(t)}{\mathrm{d} t}=\left[\varphi_{k}(t)\left(\frac{\alpha\left(K-\epsilon_{2}\right)}{\psi^{*} \epsilon_{2}+\alpha+\mu}-\epsilon_{2}\right)-(\mu+\tau)\right] y_{2}(t), \quad t \neq t_{k}, k \in \mathbb{N}, \\
y_{2}\left(t^{+}\right)=\left(1-b_{k}\right) y_{2}(t), \quad t=t_{k}, k \in \mathbb{N}, \\
y_{2}\left(t_{0}^{+}\right)=E_{0}+I_{0}>0 .
\end{array}\right.
$$


By solving above impulsive differential equation, we can obtain that for $t_{i-1}+n \omega<t<t_{i}+n \omega,(n \geq$ $\left.N_{1}\right), i=1,2, \cdots q$,

$$
\begin{aligned}
y_{2}(t)= & \left(E_{0}+I_{0}\right) \Sigma^{n} \times\left(\prod_{j=1}^{i-1}\left(1-b_{j}\right)\right) \\
& \times \exp \left[\left(\frac{\alpha\left(K-\epsilon_{2}\right)}{\psi^{*} \epsilon_{2}+\alpha+\mu}-\epsilon_{2}\right)\left(\sum_{j=1}^{i-1} \int_{t_{j-1}}^{t_{j}} \varphi_{j}(t) \mathrm{d} t+\int_{t_{i-1}+n \omega}^{t} \varphi_{j}(t) \mathrm{d} t\right)-(\mu+\tau)\left(t-t_{0}-n \omega\right)\right] .
\end{aligned}
$$

Thus, from (13) and (16), we have

$$
y_{2}(t) \rightarrow+\infty \text {, as } n \rightarrow+\infty,
$$

that is, as $t \rightarrow+\infty$, we have $y_{2}(t) \rightarrow+\infty$. By the comparison theorem, we have $\lim _{t \rightarrow+\infty}(E(t)+I(t))=+\infty$, which is a contradiction to $0<E(t)+I(t)<\epsilon_{2}$. Thus the claim is proved.

By the claim, we need to discuss the following two possible cases:

Case 1. $E(t)+I(t)>\epsilon_{2}$ for all large $t$;

Case 2. $E(t)+I(t)$ oscillates about $\epsilon_{2}$ for all large $t$.

The first case implies that the result holds. Then we will consider the second possibility. At first, set $\underline{t}$ and $\bar{t}>t^{3}$ be large enough such that

$$
E(\underline{t})+I(\underline{t}) \geq \epsilon_{2}, \quad E(\bar{t})+I(\bar{t})=\epsilon_{2}, \quad \text { and } \quad E(t)+I(t)<\epsilon_{2}, \text { for } t \in(\underline{t}, \bar{t}) .
$$

There are two possible subcases for $t$.

Subcase (I). If $\underline{t}=t_{i}+n \omega$ ( $n$ is a nonnegative integer and $i=1, \cdots, q$ ), then $E(\underline{t})+I(\underline{t})>\epsilon_{2}$ and $\left(1-b_{i}\right) \epsilon_{2}<\left(1-b_{i}\right)(E(\underline{t})+I(\underline{t})) \leq E\left(\underline{t}^{+}\right)+I\left(\underline{t}^{+}\right)<\epsilon_{2}$, where $b_{i}$ is defined in (15). We claim that there must exist a positive constant $m$, such that $E(t)+I(t) \geq m$, for $t \in(\underline{t}, \bar{t})$. Then, we will consider two possibilities in terms of the size of $\underline{t}$ and $\bar{t}$.

(i) If $\bar{t}-\underline{t} \leq n^{*} \omega$, where $n^{*}$ is defined in (14), then from system (1), we have

$$
\left\{\begin{aligned}
\frac{\mathrm{d} E(t)}{\mathrm{d} t}+\frac{\mathrm{d} I(t)}{\mathrm{d} t} & =g(t, S, E)+f(t, S, I)-\mu(E(t)+I(t))-\tau I(t) \\
& \geq-(\mu+\tau)(E(t)+I(t)), \quad t \neq t_{k}, \\
E\left(t^{+}\right)+I\left(t^{+}\right) & \geq\left(1-b_{k}\right)(E(t)+I(t)), \quad t=t_{k} .
\end{aligned}\right.
$$

From (17), we get

$$
\begin{aligned}
E(t)+I(t) & \geq\left[\prod_{i=1}^{q}\left(1-b_{i}\right)\right]^{n^{*}+1}(E(\underline{t})+I(\underline{t})) \exp [-(\mu+\tau)(t-\underline{t})] \\
& \geq\left[\prod_{i=1}^{q}\left(1-b_{i}\right)\right]^{n^{*}+1} \quad \epsilon_{2} \exp \left[-(\mu+\tau) n^{*} \omega\right] \triangleq m \quad \text { for all } t \in[\underline{t}, \bar{t}] .
\end{aligned}
$$

(ii) If $\bar{t}-\underline{t} \geq n^{*} \omega$, in view of the discussion in $(i)$, we have $E(t)+I(t) \geq m$ for all $t \in\left[\underline{t}, \underline{t}+n^{*} \omega\right]$. Next, we show that $E(t)+I(t) \geq m$ for all $t \in\left(\underline{t}+n^{*} \omega, \bar{t}\right]$. Otherwise, there exists a constant $t^{*}>0$ such that

$$
\begin{aligned}
& E(t)+I(t) \geq m, \quad \text { for all } t \in\left[\underline{t}, \underline{t}+t^{*}+n^{*} \omega\right), \\
& E\left(\underline{t}+t^{*}+n^{*} \omega\right)+I\left(\underline{t}+t^{*}+n^{*} \omega\right) \geq m, \text { and } E(t)+I(t)<m, \quad \text { for } 0<t-\left(\underline{t}+t^{*}+n^{*} \omega\right) \ll 1 .
\end{aligned}
$$

Next, we discuss two possibilities separately:

(a) For all $k \in \mathbb{N}, \underline{t}+t^{*}+n^{*} \omega \neq t_{k}$. 
It is easy to see system (15) holds on $\left[\underline{t}+n^{*} \omega, \bar{t}\right]$. So we can choose a proper $\rho>0$, such that $E\left(\underline{t}+n^{*} \omega+t^{*}\right)+I\left(\underline{t}+n^{*} \omega+t^{*}\right) \geq \rho\left(E_{0}+I_{0}\right) \geq m$. By the comparison theorem we have $0<$ $t-\left(\underline{t}+n^{*} \omega+t^{*}\right) \ll 1$,

$$
\begin{aligned}
E(t)+I(t) & \geq\left(E\left(\underline{t}+t^{*}+n^{*} \omega\right)+I\left(\underline{t}+t^{*}+n^{*} \omega\right)\right) \\
& \times \exp \left[\left(\frac{\alpha\left(K-\epsilon_{2}\right)}{\psi^{*} \epsilon_{2}+\alpha+\mu}-\epsilon_{2}\right)-(\mu+\tau)\left(t-\left(\underline{t}+n^{*} \omega+t^{*}\right)\right)\right] \\
& \geq \rho\left(E_{0}+I_{0}\right) \exp \left[\left(\frac{\alpha\left(K-\epsilon_{2}\right)}{\psi^{*} \epsilon_{2}+\alpha+\mu}-\epsilon_{2}\right)-(\mu+\tau)\left(t-\left(\underline{t}+n^{*} \omega+t^{*}\right)\right)\right] .
\end{aligned}
$$

In addition, (13) implies that

$$
\exp \left[\left(\frac{\alpha\left(K-\epsilon_{2}\right)}{\psi^{*} \epsilon_{2}+\alpha+\mu}-\epsilon_{2}\right)-(\mu+\tau)\left(t-\left(\underline{t}+n^{*} \omega+t^{*}\right)\right)\right] \geq 1,
$$

then we obtain that

$$
E(t)+I(t) \geq \rho\left(E_{0}+I_{0}\right) \exp \left[\left(\frac{\alpha\left(K-\epsilon_{2}\right)}{\psi^{*} \epsilon_{2}+\alpha+\mu}-\epsilon_{2}\right)-(\mu+\tau)\left(t-\left(\underline{t}+n^{*} \omega+t^{*}\right)\right)\right] \geq \rho\left(E_{0}+I_{0}\right) \geq m .
$$

Then $E(t)+I(t) \geq m$, for $0<t-\left(\underline{t}+t^{*}+n^{*} \omega\right) \ll 1$, which is a contradiction. Therefore, $E(t)+I(t) \geq m$ for any $t \in[\underline{t}, \bar{t}]$.

(b) There exists a $k \in \mathbb{N}$ such that $\underline{t}+t^{*}+n^{*} \omega=t_{k}$. The proof of (b) is similar to $(a)$, so we omit it. Subcase (II). If for all $k \in \mathbb{N}, \underline{t} \neq t_{k}$, then $E(\underline{t})+I(\underline{t})=\epsilon_{2}$. Using the same methods of Subcase (I), we can easily get a positive constant $m$, such that $E(t)+I(t) \geq m$, for all $t \in[\underline{t}, \bar{t}]$.

Thus, we see that $E(t)+I(t) \geq m$ for any $t \in[\underline{t}, \bar{t}]$. Since this kind of interval $[\underline{t}, \bar{t}]$ is chosen in an arbitrary way, we conclude that $E(t)+I(t) \geq m$ for all large $t$.

According to our above discussion, the choice of $m$ is independent of the positive solution of system (1), and we have proved that any solution of system (1) satisfies $E(t)+I(t) \geq m$ for sufficiently large $t$, that is, $\liminf _{t \rightarrow+\infty} E(t)+I(t) \geq m$. It is easy to obtain that, there exist positive constants $S_{*}$ such that $\liminf _{t \rightarrow+\infty} S(t) \geq S_{*}$. Therefore, the permanence of system (1) is proved.

\section{$5 \quad$ Numerical Simulations}

In this section, we will give an example for bilinear periodic incidence function to show the usefulness of the results. Rewriting the original system (1):

$$
\left\{\begin{array}{l}
\frac{\mathrm{d} S(t)}{\mathrm{d} t}=\alpha(K-S(t)-E(t)-I(t))-d_{k}(t) S(t) E(t)-c_{k}(t) S(t) I(t)-\mu S(t), \\
\frac{\mathrm{d} E(t)}{\mathrm{d} t}=d_{k}(t) S(t) E(t)+c_{k}(t) S(t) I(t)-\mu E(t)-\sigma E(t), \\
\frac{\mathrm{d} I(t)}{\mathrm{d} t}=\sigma E(t)-\mu I(t)-\tau I(t), \\
\left.\begin{array}{l}
S\left(t^{+}\right)=S(t), \\
E\left(t^{+}\right)=\left(1-p_{k}\right) E(t), \\
I\left(t^{+}\right)=\left(1-\theta_{k}\right) I(t),
\end{array}\right\} t=t_{k},
\end{array}\right\} \neq t_{k},
$$

where $\alpha, K, \mu, \sigma$ and $\tau$ are positive constants. Because symptomatic trees may be more attractive for the vectors than the asymptomatic trees [16], then we assume that $d_{k}(t) \leq c_{k}(t)$. According to Theorem 1 and Theorem 2 , we get $R_{1}$ and $R_{2}$ corresponding to system (18) as follows:

$$
R_{1}=\frac{\frac{\alpha K}{\alpha+\mu} \sum_{i=1}^{q} \int_{t_{i-1}}^{t_{i}} c_{i}(t) \mathrm{d} t}{\mu \omega-\sum_{i=1}^{q} \ln \left(1-a_{i}\right)}, \quad R_{2}=\frac{\frac{\alpha K}{\alpha+\mu} \sum_{i=1}^{q} \int_{t_{i-1}}^{t_{i}} d_{i}(t) \mathrm{d} t}{(\mu+\tau) \omega-\sum_{i=1}^{q} \ln \left(1-b_{i}\right)} .
$$


Here, we give numerical simulations of system (18) by using Matlab to illustrate the previous results. First we fix the parameters as in Table 1. In addition, from the first and second rows of data of Table 2 ,

Table 1. Parameters and initial data chosen for the simulation.

\begin{tabular}{|c|c|c|}
\hline Variable and parameter & Description & Initial or parameter values \\
\hline \multicolumn{3}{|l|}{ Variables } \\
\hline$S$ & Susceptible population & 0.7 \\
\hline$E$ & asymptomatic population & 0.15 \\
\hline$I$ & symptomatic population & 0.05 \\
\hline \multicolumn{3}{|l|}{ Parameters } \\
\hline$K$ & Maximum plant population size & 1 \\
\hline$\alpha$ & Replant rate & 0.03 \\
\hline$\mu$ & The natural death rate of citrus & 0.04 \\
\hline$\sigma$ & The transformation rate of infected trees from $\mathrm{E}$ to I & 0.02 \\
\hline$\tau$ & The HLB-induced death rate & 0.025 \\
\hline$\omega$ & pulse period & 12 \\
\hline$q$ & pulse times in a period & 4 \\
\hline$c_{1}(t)$ & - & $0.1+0.03 \sin ((\pi t) / 6)$ \\
\hline$c_{2}(t)$ & - & $0.05+0.02 \sin ((\pi t) / 6)$ \\
\hline$c_{3}(t)$ & - & $0.3+0.05 \sin ((\pi t) / 6)$ \\
\hline$c_{4}(t)$ & - & $0.25+0.05 \sin ((\pi t) / 6)$ \\
\hline$d_{1}(t)$ & - & $0.05+0.03 \sin ((\pi t) / 6)$ \\
\hline$d_{2}(t)$ & - & $0.025+0.02 \sin ((\pi t) / 6)$ \\
\hline$d_{3}(t)$ & - & $0.15+0.05 \sin ((\pi t) / 6)$ \\
\hline$d_{4}(t)$ & - & $0.125+0.05 \sin ((\pi t) / 6)$ \\
\hline
\end{tabular}

we can compute the value of $R_{1}$ or $R_{2}$ and obtain the effect of parameters $\theta_{k}, p_{k}$ on the extinction and permanence of the disease.

Table 2. The effect of pulse control strength on the extinction and permanence of the disease.

\begin{tabular}{|l|l|l|l|l|l|l|l|l|l|l|}
\hline parameter & $\theta_{1}$ & $\theta_{2}$ & $\theta_{3}$ & $\theta_{4}$ & $p_{1}$ & $p_{2}$ & $p_{3}$ & $p_{4}$ & $R_{1}\left(R_{2}\right)$ & disease \\
\hline value & 0.15 & 0.52 & 0.53 & 0.2 & 0.12 & 0.52 & 0.53 & 0.2 & $R_{1}=0.9965$ & extinction \\
\hline value & 0.15 & 0.14 & 0.15 & 0.2 & 0.12 & 0.12 & 0.13 & 0.2 & $R_{2}=1.0045$ & permanence \\
\hline
\end{tabular}

Fig. 1 and Fig. 2 show the the disease will die out when $R_{1}<1$ and will be endemic when $R_{2}>1$, respectively.

Next, we use controlling variables method to study the impact of parameter $\alpha$ and $\tau$ on $R_{1}$ and $R_{2}$, respectively. Let $\alpha$ vary in $[0,1]$ with the other parameters unchanged, we get the graph for $R_{1}$ to $\alpha$ (see Fig. 3). It shows that $R_{1}$ increases as $\alpha$ increases from 0 to 1 , and $R_{1}$ is more sensitive when $\alpha<0.1$. If we vary $\tau$ in [0,1] in (18), $R_{2}$ decreases as $\tau$ increases from 0 to 1 , and $R_{2}$ is more sensitive when $\tau<0.3$ (see Fig. 4). If we take parameters as $\theta_{1}=0.15, \theta_{2}=0.38, \theta_{3}=0.42, \theta_{4}=0.2, p_{1}=0.12, p_{2}=0.33, p_{3}=0.38$, $p_{4}=0.2$, with the other parameters unchanged as previously defined, numerical calculations indicate that 


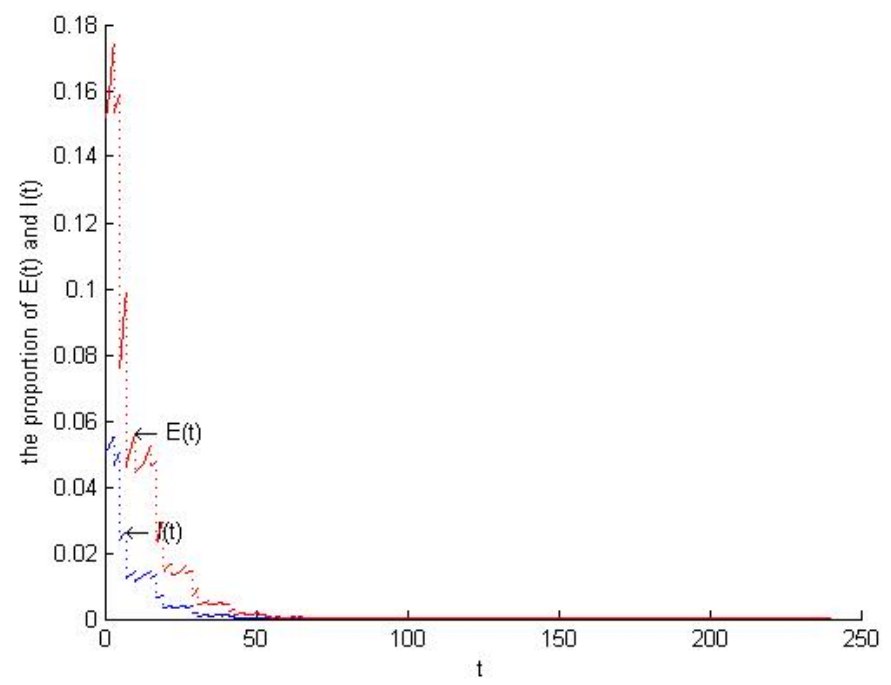

Figure 1. This figure shows the movement path of $I$ and $E$, as functions of time $t$, where $\theta_{1}=0.15, \theta_{2}=0.52$, $\theta_{3}=0.53, \theta_{4}=0.2, p_{1}=0.12, p_{2}=0.52, p_{3}=0.53, p_{4}=0.2$. The disease will be extinct eventually $\left(R_{1}=0.9965<1\right)$.

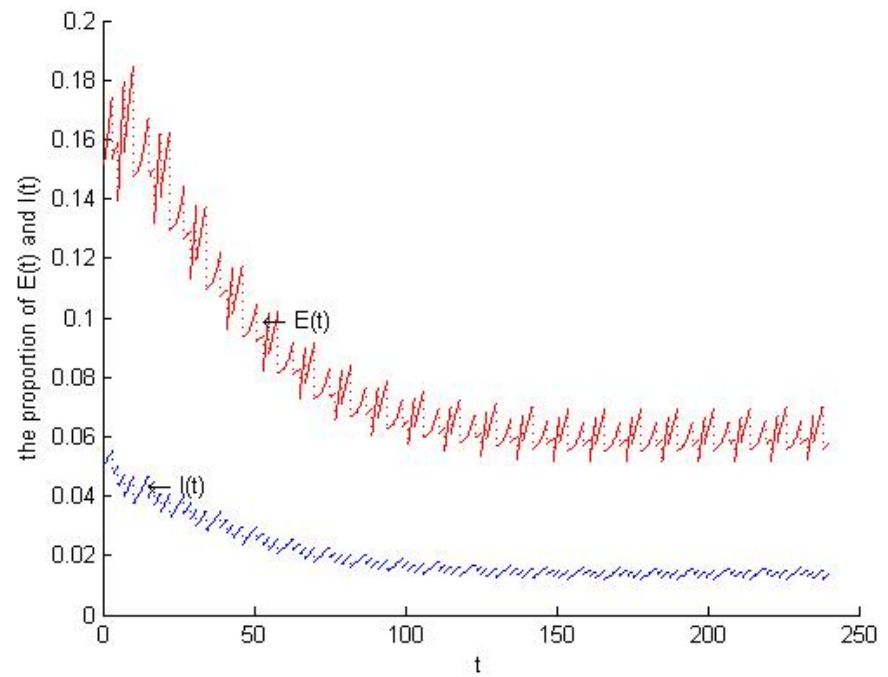

Figure 2. This figure shows the movement path of $I$ and $E$, as functions of time $t$, where $\theta_{1}=0.15, \theta_{2}=0.14$, $\theta_{3}=0.15, \theta_{4}=0.2, p_{1}=0.12, p_{2}=0.12, p_{3}=0.13, p_{4}=0.2$. the disease is permanent $\left(R_{2}=1.0045>1\right)$.

the the disease will fade out (see Fig. 5), whereas we can compute $R_{1}=1.3943>1$. Moreover, if we take $\theta_{1}=0.15, \theta_{2}=0.3, \theta_{3}=0.2, \theta_{4}=0.2, p_{1}=0.12, p_{2}=0.25, p_{3}=0.1, p_{4}=0.2$, numerical calculations indicate that the the disease will persist (see Fig. 6), whereas $R_{2}=0.8007<1$. Thus, the conditions of Theorem 1 and Theorem 2 are only sufficient, not necessary. That is to say, using $R_{1}\left(R_{2}\right)$ as the extinction (permanence) threshold of the disease will overestimate or underestimate the disease transmission risk. 


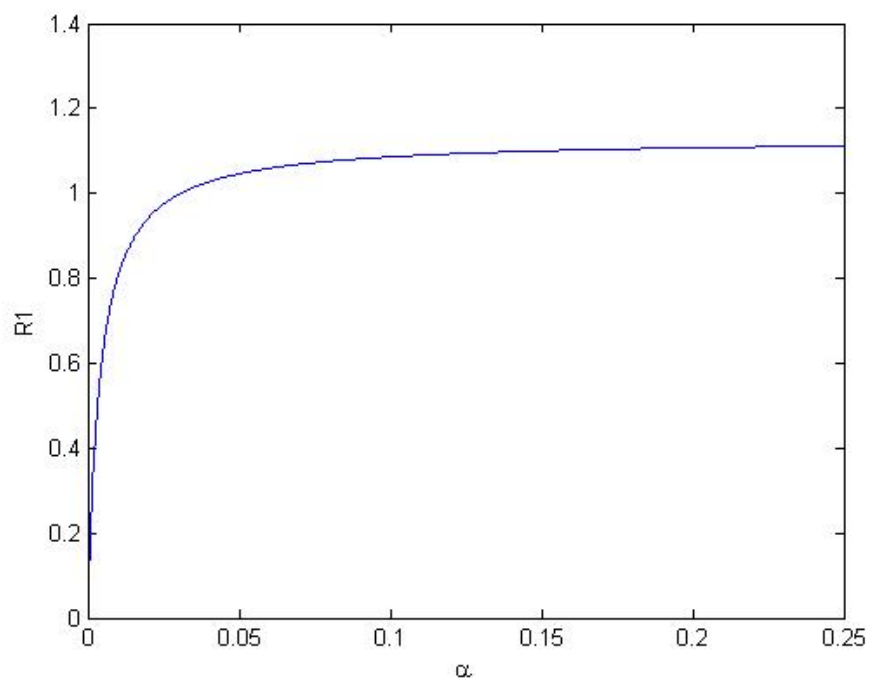

Figure 3. The graph of $R_{1}$ versus $\alpha$.

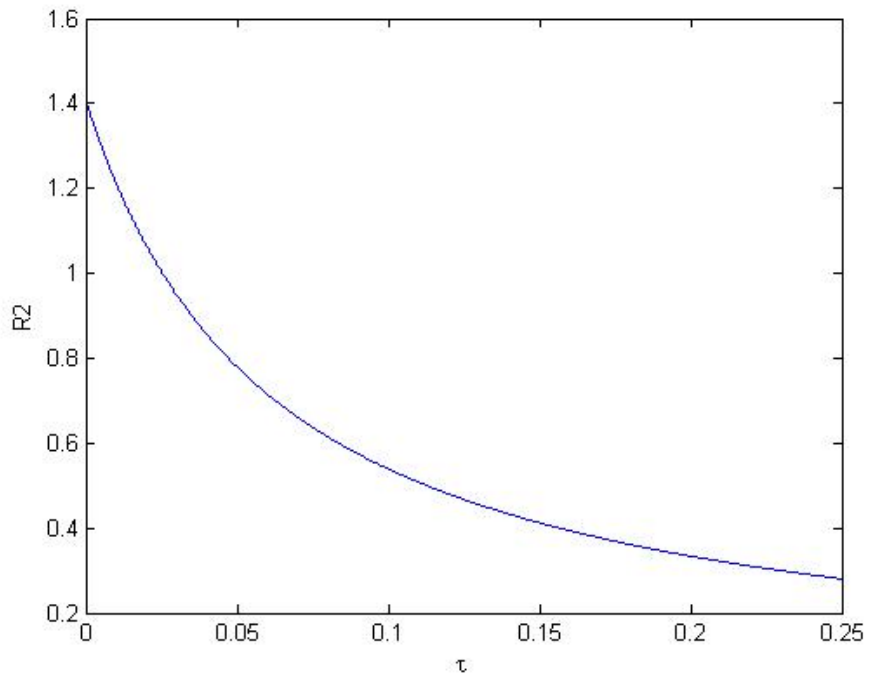

Figure 4. The graph of $R_{2}$ versus $\tau$.

\section{Conclusion}

In this paper, the model developed describes the population dynamics for citrus HLB with general incidence, in which varying multi-pulse within an environmental period is considered. We show that when $R_{1}<1$, the disease-free periodic solution is globally attractive, whereas when $R_{2}>1$, the disease is uniformly persistent. The simulation indicates that: (i) pulse roguing infected citrus trees is an effective method for HLB control, (ii) increasing the replanting rate is bad for HLB control, (iii) increasing HLBinduced death rate is benefit for HLB control. However, our results can not solve the critical threshold value which determines the extinction and the uniform persistence of disease. Additionally, our model may be simple, the vector population (citrus psyllid) is not considered, which indicates that our model and results have a lot of room to improve. 
Table 3. The effect of pulse control strength on the extinction and permanence of the disease.

\begin{tabular}{|l|l|l|l|l|l|l|l|l|l|l|}
\hline parameter & $\theta_{1}$ & $\theta_{2}$ & $\theta_{3}$ & $\theta_{4}$ & $p_{1}$ & $p_{2}$ & $p_{3}$ & $p_{4}$ & $R_{1}\left(R_{2}\right)$ & disease \\
\hline value & 0.15 & 0.38 & 0.42 & 0.2 & 0.12 & 0.33 & 0.38 & 0.2 & $R_{1}=1.3943$ & extinct \\
\hline value & 0.15 & 0.3 & 0.2 & 0.2 & 0.12 & 0.25 & 0.1 & 0.2 & $R_{2}=0.8007$ & persistent \\
\hline
\end{tabular}

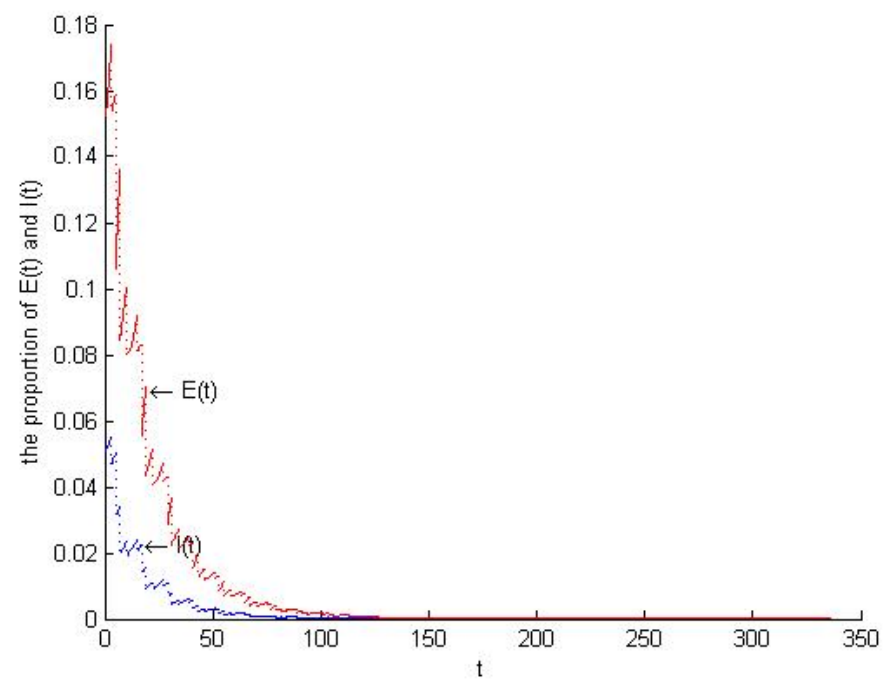

Figure 5. This figure shows the movement path of $I$ and $E$, as functions of time $t . R_{1}=1.3943>1$.

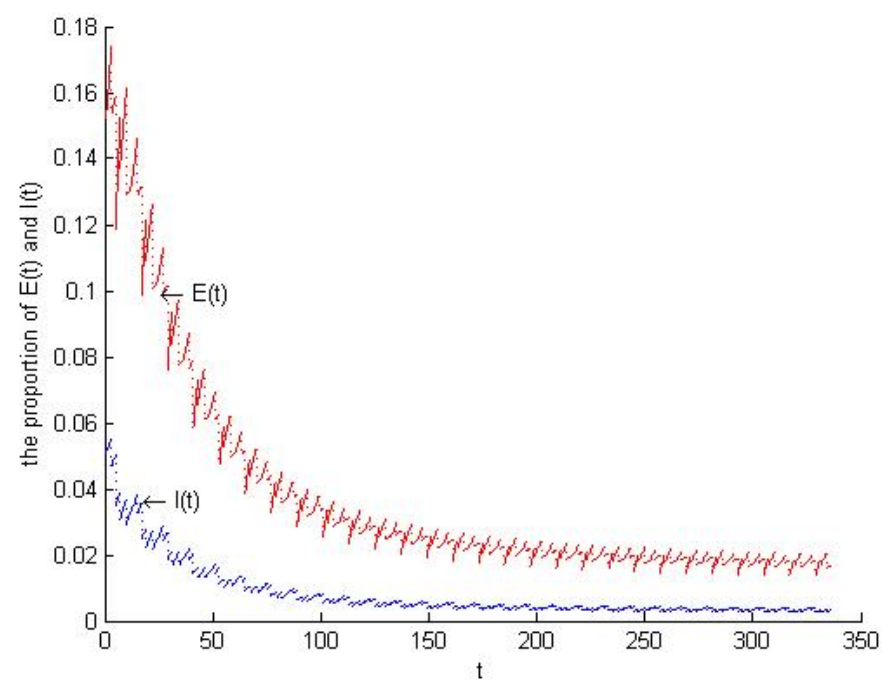

Figure 6. This figure shows the movement path of $I$ and $E$, as functions of time $t . R_{2}=0.8007<1$.

Acknowledgments. The research has been supported by the Natural Science Foundation of China (No. 11261004, 11561004), the Natural Science Foundation of Jiangxi Province (No. 20151BAB201016), and the Postgraduate Innovation Fund of Jiangxi Province (No. YC2015-S375). 


\section{References}

1. J. M. Bov, "Huanglongbing: A destructive, newly-emerging, century-old disease of citrus," J. Plant Pathol, vol. 88, no. 1, pp. 7-37, 2006.

2. CISR: Leading the Fight Against Invasive Species in California. [Online]. Available: http://cisr.ucr.edu/citrus\% 20greening.html

3. CISR Blog: Huanglongbing Detected in Hacienda Heights, Los Angeles County. [Online]. Available: http://cisr.ucr.edu/blog/invasive-species/huanglongbing-detected-in-hacienda-heights-los-angeles-county/

4. C. Chiyaka and B. H. Singer, "Modeling huanglongbing transmission within a citrus tree," in Proceedings of the National Academy of Sciences of the United States of America, vol. 109, no. 30, pp. 12213-12218, 2012.

5. H. D. Coletta-Filho, M. P. Daugherty, C. Ferreira and J. R. Lopes, "Temporal progression of "Candidatus liberibacter asiaticus' infection in citrus and acquisition efficiency by diaphorina citri," Phytopathology, vol. 104, no. 4, pp. 416-421, 2014.

6. R. d'A. Vilamiu, S. Ternes, A. Braga. and F. Laranjeira, "A model for Huanglongbing spread between citrus plants includingdelay times and human intervention," AIP Conference Proceedings, vol. 1479, no. 1, pp. 2315-2319, 2012

7. R. d'A. Vilamiu, S. Ternes, A. Braga and F. Laranjeira, "Modelling the effect of an alternative host population on the spread of citrus Huanglongbing," AIP Conference Proceedings, vol. 1558, no. 1, pp. 2504-2508, 2013.

8. S. Gao and J. Wang, "Threshold dynamics of a Huanglongbing model with Logistic growth in periodic environments," Abstract and Applied Analysis, vol. 2014, Special, 2013.

9. K. Jacobsen, J. Stupiansky and S. S. Pilyugin, "Mathematical modeling of citrus groves infected by huanglongbing," Mathematical Biosciences and Engineering, vol. 10, no. 3, pp. 705-728, 2013.

10. J. Gao, B. Shen, E. Feng and Z. Xiu, "Modelling and optimal control for an impulsive dynamical system in microbial fed-batch culture," Computational and Applied Mathematics, vol. 32, no. 2, pp. 275-290, 2013.

11. V. Kuzovkin, V. Filatov and M. Chumaeva, "Nonlinear models of dynamic processes in impulse control systems of brushless DC motors," Journal of Computer and Systems Sciences International, vol. 53, no. 3, pp. 420-429, 2014.

12. T. Zhang, X. Meng, R. Liu and T. Zhang, "Periodic solution of a pest management Gompertz model with impulsive state feedback control," Nonlinear Dynamics, vol. 78, no. 2, pp. 921-938, 2014.

13. J. Liu, T. Zhang and J. Lu, "An impulsive controlled eco-epidemic model with disease in the prey," Journal of Applied Mathematics and Computing, vol. 53, no. 1-2, pp. 459-475, 2012.

14. X. Ji, S. Yuan and L. Chen, "A pest control model with state-dependent impulses," International Journal of Biomathematics, vol. 8, no. 1, pp. 155009, 2015.

15. X. Xu, Y. Xiao and R. Cheke, "Models of impulsive culling of mosquitoes to interrupt transmission of West Nile virus to birds," Applied Mathematical Modelling, vol. 39, no. 13, pp. 3549-3568, 2014.

16. D. G. Hall and M. G. Hentz, "A comparison of traps and stem tap sampling for monitoring adult Asian citrus psyllid (Hemiptera: Psyllidae) in citrus," Fla. Entomol, vol. 90, no. 2, pp. 327-334, 2007. 